\title{
SNPing away at innate immunity
}

\author{
Stephen J. Chanock and Charles B. Foster
}

Commentary

See related article

this issue, pages 431-437.

Immunocompromised Host Section, Pediatric Oncology Branch, National Cancer Institute, National Institutes of Health, Bethesda, Maryland, 20892 USA

Address correspondence to: Stephen Chanock, Immunocompromised Host Section, Pediatric Oncology Branch, Advanced Technology Center, National Cancer Institute, 8718 Grovemont Circle, Gaithersburg, Maryland 20877, USA. Phone: (301) 435-7559; Fax: (301) 402-3134; E-mail: sc83a@nih.gov.

Cystic fibrosis (CF) is a genetic disorder caused by mutation in the cystic fibrosis transmembrane conductance regulator (CFTR) (1). A hallmark of the disease is a defect in chloride conductance, particularly in serous gland cells and respiratory epithelial cells. Depletion of the periciliary liquid layer, partly due to a defect in ciliary transport, has pleiotropic consequences with respect to local host defenses against bacterial infection, especially by Pseudomonas aeruginosa (2). The surface fluid, covering airway epithelia in CF, has been shown to have deficient bactericidal activity. Because the defect reverses when the $\mathrm{NaCl}$ concentration is decreased, it is hypothesized that the high salt concentration in CF pulmonary secretions could inactivate antimicrobial molecules of the innate immune system, such as defensins, surfactant proteins, lysozyme, and, perhaps, mannose-binding lectin (MBL) (3-5). A small difference in either the function or level of 1 or more molecules of innate immunity could influence the host's ability to control pathogenic bacteria. Despite having an intense inflammatory response and an intact adaptive immune response, most CF patients eventually become chronically colonized with $P$. aeruginosa, considered to be causally related to the steady deterioration in pulmonary function. Of particular clinical concern is colonization by Burkholderia cepacia, which leads to severe, recurrent pulmonary problems (6).

In this issue of the JCI, Garred et al. have shown that common genetic variations in the mannose-binding lectin gene $(M B L 2)$ are associated with increased disease severity and a higher risk of infection by B. cepacia in patients with CF (7). The presence of MBL variant alleles is also associated with poor prognosis and early death. The wildtype human MBL is synthesized in the liver as an acute-phase serum protein, and occupies a central position in the innate immune system. MBL is capable of opsonizing pathogenic microorganisms and, in turn, activating the complement system $(8,9)$. Although the role of MBL in the lung is not well understood, it is possible that MBL is an important member of pulmonary host defenses and contributes to the initial response by invading organisms. Furthermore, MBL could directly or indirectly influence the influx or clearing of neutrophils in the respiratory lumen. In the setting of CF, it was reasonable to consider that the effect of an MBL variant could be magnified against the background of CF lung disease. Common polymorphisms in both the regulatory and structural regions of the gene result in marked variation in circulating levels of MBL $(10,11)$. Thus, it is not surprising that the authors correlated levels of $\mathrm{MBL}$, lung function, and median survival with different genotypes of $M B L 2$. This study provides strong evidence supporting the hypothesis that perturbations of innate immunity contribute to the pathogenesis of lung disease in CF.

The clinical importance of genetic variants of MBL to both infectious and inflammatory disorders has been demonstrated in previous association studies (9). The current study, along with several others, suggests that the impact of MBL deficiency might be magnified in individuals with either a chronic infection or an underlying disruption of immune function. Previous examples include the association of variant MBL alleles with susceptibility to HIV infection and progression of AIDS; with systemic lupus erythematosus; and with chronic hepatitis B infection (12-14). The risk for developing autoimmune complications of chronic granulomatous disease (CGD), a rare primary immune deficiency, is increased in individuals with variant alleles of MBL (15). In CGD, a variant genotype of the FcyRIIa receptor (another key component in innate immunity) was also associated with autoimmunity. Results such as these suggest that association studies can now help to identify secondary genes that modify outcome in primary genetic disorders. The identification of a collection of modifying genotypes could lead to a more refined understanding of a specific disease. Understanding the role of modifying genes has considerable potential for determining risk profiles that might impact therapeutic decisions. By subclassifying diseases, according to secondary genetic risk factors, therapy could be adapted to individual needs. Indeed, the current study raises the possibility that recombinant or purified MBL may be of benefit to at least a subset of CF patients.

Because the associated phenotype of a common polymorphism in innate immunity is often subtle, studying common genetic variants in the normal host may be exceedingly difficult. More than $35 \%$ of the general population has at least 1 structural variant allele of MBL2 (9-11). Even within a disease population such as CF, the overall distribution of the variant allele does not differ from healthy controls. However, when variant alleles are examined closely within a population of CF patients, clear relationships are observed between variant genotypes and deleterious outcome. In general, disease populations, such as those in which major defects in host defenses are observed, may greatly enhance our ability to observe the consequences of subtle genetic variations in innate immunity. Armed with an expanding number of immunologically important polymorphisms, genetic association studies should proceed with an eye toward understanding immunological pathways in vivo.

Until recently, the ability to perform genetic association studies has been limited by the paucity of known polymorphisms. However, advances in 
high-throughput sequence analysis and DNA chip arrays have begun to generate a larger and more comprehensive set of genetic reagents for understanding the relationship between genetics and disease $(16,17)$. As the Human Genome Project progresses toward completion early in the next millennium, a catalog of known genes and their variations in sequence has already begun to take shape (18). Types of DNA sequence variations include insertions, deletions, variable numbers of repeat sequences, and, the most common, single-nucleotide polymorphisms (SNPs). Although the project is still far from complete, estimates of the number of SNPs vary between 1 in 300 to 1 in 1200 bp (19). While the final frequency will not be known for some time, the rich diversity in the human genome promises to provide a valuable resource for understanding individual differences in disease expression.

To this end, the National Cancer Institute's Genetic Annotation Initiative (20) a publicly available resource, has launched a program to resequence and identify SNPs within several thousand candidate genes. In the near future, it will be possible to systematically study the contribution of candidate polymorphisms selected from a catalog of genes, many of which had previously exhibited 1 or more biological or clinical associations. Pursuing this path, further investigation of CF pulmonary disease could well identify candidate genes encoding proteins that influence apoptosis, proinflammatory cytokines, myeloid-specific proteins, surfactant proteins, collectins, or the Th1/Th2 cytokines to the detriment of the patient.

Genetic annotation studies offer a new way to view the dynamic relationship between genes and their products in vivo. Identifying and cataloging physiologically important SNPs, however, is only part of the challenge. Meaningful association studies require high-quality, detailed epidemiological data. Furthermore, sophisticated informatics will be needed to handle the volume of data and provide a resource for the daunting challenge of statistically analyzing sets of genes for their effect on different outcomes. Positive association between 1 or more genotypes and different outcomes will need to be validated in a series of studies. Assessing risk and applying the conclusions to clinical decision making will require careful consideration of complex ethical questions as well.

Using the candidate gene approach, Garred et al. have identified a modifying gene in CF, namely variants of $M B L 2$, and at the same time have provided further evidence that the pathogenesis of $\mathrm{CF}$ is related to perturbations in innate immunity. Before we can test all CF patients at this locus and confidently tailor therapy according to $M B L 2$ variant genotypes, there is much work to be done. The study must be confirmed in other populations of CF patients. The findings of this genetic annotation study should stimulate other investigators to examine the biological role of MBL in pulmonary host defenses. Moreover, the authors have shown that a genetic disease like CF is not as simple as originally envisioned. The cloning of the CFTR was a major milestone in understanding the molecular basis of $\mathrm{CF}$, but dissecting the pathogenesis of CF has proved far more complicated (1). Even among patients with the most common CFTR mutation, $\Delta F 508$, the course of lung disease differs markedly (21). The challenge remains to select appropriate candidate SNPs in order to achieve a more complete understanding of the pathogenesis of a disease as complex as CF.

1. Riordan, J.R., et al. 1989. Identification of the cystic fibrosis gene: cloning and characterization of complementary DNA. Science. 245:1066-1073.

2. Matsui, H., et al. 1998. Evidence for periciliary liquid layer depletion, not abnormal ion composition, in the pathogenesis of cystic fibrosis airways disease. Cell. 95:1005-1015

3. Bals, R., et al. 1998. Human beta-defensin 2 is a salt-sensitive peptide antibiotic expressed in human lung. J. Clin. Invest. 102:874-880.
4. Goldman, M.J., et al. 1997. Human betadefensin- 1 is a salt-sensitive antibiotic in lung that is inactivated in cystic fibrosis. Cell. 88:553-560.

5. Smith, J.J., Travis, S.M., Greenberg, E.P., and Welsh, M.J. 1996. Cystic fibrosis airway epithelia fail to kill bacteria because of abnormal airway surface fluid [erratum 1996, 87:355]. Cell. 85:229-236.

6. Govan, J.R., and Deretic, V. 1996. Microbial pathogenesis in cystic fibrosis: mucoid Pseudomonas aeruginosa and Burkholderia cepacia. Microbiol Rev. 60:539-574.

7. Garred, P., et al. 1999. Association of mannosebinding lectin gene heterozygosity with severity of lung disease and survival in cystic fibrosis. J. Clin. Invest 104:431-437.

8. Epstein, J., Eichbaum, Q., Sheriff, S., and Ezekowitz, R.A. 1996. The collectins in innate immunity. Curr. Opin. Immunol. 8:29-35.

9. Babovic-Vuksanovic, D., Snow, K., and Ten, R.M. 1999. Mannose-binding lectin (MBL) deficiency. Variant alleles in a midwestern population of the United States. Ann. Allergy Asthma Immunol. 82:134-138, 141; quiz 142-143.

10. Madsen, H.O., et al. 1994. A new frequent allele is the missing link in the structural polymorphism of the human mannan-binding protein. Immunogenetics. 40:37-44.

11. Madsen, H.O., et al. 1995. Interplay between promoter and structural gene variants control basal serum level of mannan-binding protein. $J$ Immunol. 155:3013-3020.

12. Garred, P., et al. 1997. Susceptibility to HIV infection and progression of AIDS in relation to variant alleles of mannose-binding lectin. Lancet. 349:236-240.

13. Davies, E.J., et al. 1995. Mannose-binding protein gene polymorphism in systemic lupus erythematosus [see comments]. Arthritis Rheum. 38:110-114.

14. Thomas, H.C., et al. 1996. Mutation of gene of mannose-binding protein associated with chronic hepatitis B viral infection [see comments]. Lancet. 348:1417-1419.

15. Foster, C.B., et al. 1998. Host defense molecule polymorphisms influence the risk for immunemediated complications in chronic granulomatous disease. J. Clin. Invest. 102:2146-2155.

16. Wang, D.G., et al. 1998. Large-scale identification, mapping, and genotyping of singlenucleotide polymorphisms in the human genome. Science. 280:1077-1082.

17. Gilles, P.N., Wu, D.J., Foster, C.B., Dillon, P.J., and Chanock, S.J. 1999. Single nucleotide polymorphic discrimination by an electronic dot blot assay on semiconductor microchips. Nat. Biotechnol. 17:365-370.

18. Collins, F.S. 1999. Shattuck lecture - medical and societal consequences of the Human Genome Project. N. Engl. J. Med. 341:28-37.

19. Cargill, M., et al. 1999. Characterization of single-nucleotide polymorphisms in coding regions of human genes. Nat. Genet. 22:231-238.

20. National Cancer Institute Genetic Annotation Initiative. http://lpg.nci.nih.gov/GAI.

21. Kerem, B., and Kerem, E. 1996. The molecular basis for disease variability in cystic fibrosis. Eur. J. Hum. Genet. 4:65-73. 